\title{
Escala de Afeto Positivo e Negativo para Crianças: Estudos de Construção e Validação
}

\author{
Escala de Afeto Positivo e Negativo para Crianças ${ }^{1,2}$
}

\author{
Claudia Hofheinz Giacomoni \\ Cláudio Simon Hutz
}

\begin{abstract}
Resumo
O objetivo deste estudo foi desenvolver uma Escala de Afeto Positivo e Negativo para Crianças. São apresentados dois estudos: de construção da escala, bem como de validação concorrente. Além disso, procurou-se avaliar o nível de afeto positivo e negativo das crianças dessa amostra, assim como, verificar possíveis diferenças entre sexos, faixa etária e tipo de escola. Participaram do estudo de construção da escala 66 I crianças (52,2\% meninos) entre sete e 12 anos ( $m=10,6$ anos; d.p. = I,7 anos) de escolas públicas estaduais (54\%) e privadas (46\%) de Porto Alegre. A versão final da Escala de Afeto Positivo e Negativo é composta por 34 itens, 17 itens em cada subescala. Os coeficientes Alpha obtidos para as subescalas de Afeto Positivo $(0,88)$ e de Afeto Negativo $(0,84)$ apontam evidências de confiabilidade. Análises Fatoriais confirmaram a estrutura da escala através da solução de dois fatores em ambos estudos. A Escala também apresentou boas evidências de validação concorrente. Através de correlações coerentes com as apontadas pela literatura, os resultados indicam que a Escala de Afeto Negativo pode ser utilizada como instrumento de avaliação de desajustamento emocional. O desenvolvimento da Escala de Afeto Positivo e Negativo para Crianças incrementa o processo de elaboração de instrumentos para avaliarmos o bemestar subjetivo infantil.
\end{abstract}

Palavras-Chave: Afeto positivo; Afeto negativo; Escala.

\section{Positive and Negative Affect Schedule for Children: Development and Validation Studies}

\begin{abstract}
The main purpose of this study was to develop a Children's Positive and Negative Affect Schedule. We presented two studies - development and validation studies. We evaluated the children's positive and negative affect level, as well possible differences between sex, age and kind of school (private $\mathrm{x}$ public). $66 \mathrm{I}$ children $(52.2 \%$ boys), age seven to 12 years $(m=10.6$ years; d.p. $=1.7)$ participated scale development' studies, from public (54\%) and private schools in Porto Alegre. The Positive and Negative Affect Schedule finalized with 34 items, 17 in each subscale. The subscales demonstrate acceptable internal consistency (Positive Affect Subscale $=0.88$; Negative Affect Subscale $=0.84$ ). The results of Factor Analysis confirmed a two factor solution. Were obtained concurrent validation. The results indicated that Children's Positive and Negative Affect Schedule correlated as expected with various criterion measures. The Children's Positive and Negative Affect Schedule appears useful for research and to develop the subjective well-being area.
\end{abstract}

Key-words: Positive affect; Negative affect; Measure.

\footnotetext{
'Pesquisa com apoio da CAPES

2 Os autores agradecem a colaboração dos alunos do Curso de Psicologia da UFRGS, Ana Paula Tibulo, Gabriel da Silva Mazzini e Joceline Fátima

Zanchetin pela participação na coleta, digitação e análise dos dados.
} 


\section{Escala de Afecto Positivo y Negativo para Niños: Estudios de Construcción y Validez}

\section{Resumen}

El objetivo de este estudio fue desarrollar una Escala de Afecto Positivo y Negativo para Niños. Son presentados dos estudios: de construcción de la escala, así como de validación concorrente. Además de eso, se ha buscado evaluar el nivel de afecto positivo y negativo de los niños de esa muestra, así como verificar posibles diferencias entre sexos, grupos de edad y tipo de escuela. La versión final de la Escala de Afecto Positivo y Negativo es compuesta por 34 ítems, 17 ítems en cada sub-escala. Los coeficientes Alpha obtenidos para las sub-escalas de Afecto Positivo $(0,88)$ y de Afecto Negativo $(0,84)$ apuntan evidencias de fiabilidad. Análisis Factoriales confirmaron la estructura de la escala a través de la solución de dos factores en los dos estudios. La Escala también presentó buenas evidencias de validación concorrente. A través de correlaciones coherentes con las apuntadas por la literatura, los resultados indican que la Escala de Afecto Negativo puede ser utilizada como instrumento de evaluación de desajuste emocional. El desarrollo de la Escala de Afecto Positivo y Negativo para Niños incrementa el proceso de elaboración de instrumentos para que evaluemos el bien estar subjetivo infantil. Palabras-Clave: Afecto positivo; Afecto negativo; Escala

\section{Introdução}

Bem-estar subjetivo é definido como a avaliação cognitiva e afetiva que a pessoa faz sobre a própria vida. Essa avaliação inclui as reações emocionais aos eventos, assim como os julgamentos cognitivos de satisfação para com a própria vida. Sendo assim, bemestar subjetivo é um conceito amplo que inclui experienciar emoções prazerosas, baixos níveis de afeto negativo e altos níveis de satisfação de vida (Diener, Lucas \& Oishi, 2005).

$O$ afeto, segundo componente do bem-estar subjetivo, é composto pelas respostas afetivas das pessoas, incluindo afetos prazerosos e desprazerosos (Andrews \& Withey, 1976; Campbell, Converse \& Rodgers, 1976; Diener, 1984, 1994; Diener \& cols, 1999). Segundo Watson, Clark e Tellegen (1988), duas dimensões consistentes emergem nos estudos sobre a estrutura afetiva ao longo das culturas: afeto positivo e afeto negativo. $O$ afeto positivo reflete o quanto uma pessoa está sentindo-se entusiástica, ativa e alerta, enquanto o afeto negativo é uma dimensão geral da angústia e insatisfação, o qual inclui uma variedade de estados de humor aversivos, incluindo raiva, culpa, desgosto, medo (Watson, Clark \& Tellegen, 1988).

Considerando-se que faz-se necessária a avaliação das duas dimensões de afeto (Lucas, Diener \& Larsen, 2003) para uma completa avaliação do bem-estar subjetivo e constatando a inexistência de medidas adequadamente elaboradas para crianças e desenvolvidas a partir de nossa cultura, o objetivo deste estudo foi desenvolver uma Escala de Afeto Positivo e Negativo para Crianças. São apresentados os estudos de construção da escala, bem como de validação concorrente. Além disso, procurou-se avaliar o nível de afeto positivo e negativo das crianças dessa amostra, assim como, verificar possíveis diferenças entre sexos, faixa etária e tipo de escola.

\section{Estudo I}

\section{Construção da Escala de Afeto Positivo e Negativo para Crianças}

Para a elaboração da escala proposta, optou-se por compor os itens através de adjetivos descritores de afeto positivo e negativo, seguindo a opção de outros pesquisadores que investigaram o afeto através de adjetivos marcadores (Giacomoni \& Hutz, 1997; Watson, Clark \& Tellegen, 1988; Laurent \& cols., 1999). Os itens-adjetivos foram selecionados a partir da análise de 200 entrevistas conduzidas sobre o conceito de felicidade e suas características em crianças de cinco a doze anos descritos no estudo de Giacomoni (2002). Além disso, também foram consultados livros da literatura infantil, aproximadamente 30 obras, com o objetivo de identificar os adjetivos utilizados com maior freqüência na literatura infantil. Também foram verificados os adjetivos finais da Escala de Afeto Positivo e Negativo para Crianças de Laurent e colaboradores (1999), com o intuito de explorar seus principais itens, entretanto, 
esses não foram meramente traduzidos e utilizados, já que a literatura de construção de instrumentos desaconselha tal prática. Inicialmente, foi elaborada uma listagem de aproximadamente 80 itens, sendo 40 positivos e 40 negativos, provenientes do estudo de Giacomoni (2002), do levantamento da literatura infantil e Escala de Afeto Positivo e Negativo para Crianças de Laurent e colaboradores (1999). Todo o processo de elaboração dos itens foi realizado por um grupo de pesquisa formado por pesquisadores em Psicologia do Desenvolvimento e por alunos de graduação em Psicologia. Após esta etapa, os itens foram analisados individualmente por cada membro da equipe de pesquisa, que elaborou um ranking dos itens considerados mais adequados e consistentes para as dimensões positiva e negativa. Com a comparação dos rankings obtidos, foram selecionados os itens que obtiveram maior predileção. Após essa seleção, os itens finais foram analisados por um juiz que possui conhecimento na área. Foram então selecionados 50 itens.

Por sua vez, esses 50 itens foram mostrados para 10 crianças entre sete e doze anos de idade. Foi utilizada uma escala de respostas do tipo Likert de 5 pontos, que variavam de I a 5, (I) nem um pouco; (2) um pouco; (3) mais ou menos; (4) bastante; (5) muitíssimo, para indicar o quanto elas estavam sentindo-se de acordo com o item. Por meio desse procedimento, foram eliminados seis itens devido ao grau de dificuldade dos mesmos. Foi comprovado o nível de compreensão da escala de resposta de cinco pontos. A versão preliminar da escala finalizou com 44 itens, sendo 18 itens positivos e 26 itens negativos. A próxima etapa realizada foi a testagem desta versão preliminar da escala.

\section{Método}

\section{Participantes}

O número de participantes desta etapa do estudo foi calculado procurando atender ao critério da "razão itens/sujeito", geralmente utilizado para o cálculo amostral quando são necessárias Análises Fatoriais. Conforme tal critério, para que se possa realizar uma
Análise Fatorial confiável é importante que a amostra contenha pelo menos cinco vezes o número de itens do instrumento a ser avaliado.

Participaram deste estudo $66 \mathrm{I}$ crianças de ambos os sexos, 345 meninos $(52,2 \%)$ e 316 meninas $(47,8 \%)$. A faixa etária variou entre sete e 12 anos $(M=10,6$ anos; $D P=1,7$ anos $)$. As crianças freqüentavam o ensino fundamental, entre a $2^{\mathrm{a}}$ e $6^{\mathrm{a}}$ séries, de escolas públicas estaduais $(54 \%)$ e privadas (46\%) de Porto Alegre. Desta amostra, 66\% das crianças moravam com ambos os pais, $31 \%$ viviam com somente um dos pais e $3 \%$ não viviam com esses. O número médio de irmãos relatados pelas crianças foi de I,5 irmãos (DP = I,30). Os dados demográficos da amostra são descritos na Tabela $\mathrm{I}$.

\section{Procedimento}

A seleção das escolas foi feita através de amostragem por área (Cozby, 1981). Inicialmente, foi feito o contato com as escolas para apresentação do projeto de pesquisa e da documentação necessária. Obtida a aprovação do projeto pela equipe técnica da escola e pelos professores, foram encaminhados para os pais ou responsáveis os termos de consentimento. $O$ presente estudo está de acordo com o Conselho Nacional de Saúde e com a Resolução n. 016/2000 do Conselho Federal de Psicologia, que versam sobre os aspectos éticos da pesquisa com seres humanos. Salvaguardou-se a todos os participantes o direito de sigilo, voluntariado e interrupção da participação. A explicação do estudo, seus objetivos e finalidades foram apresentadas de forma coletiva para as crianças na própria sala de aula. Mediante os termos assinados pelos pais e/ou responsáveis, a escala foi aplicada coletivamente na sala de aula. Durante a aplicação, a presença dos professores foi facultativa. A aplicação foi realizada pelo pesquisador coordenador e por um auxiliar de pesquisa. Com o objetivo de instruir as crianças sobre o preenchimento da escala e da correta utilização da escala tipo Likert do instrumento, as crianças recebiam as seguintes instruções, que eram acompanhadas pelo desenho da escala de respostas no quadro-negro da sala. 
Tabela I. Estatísticas descritivas dos dados demográficos da amostra por sexo, faixa etária, tipo de escola e série

\begin{tabular}{llrc}
\hline Variável & & $n$ & $\%$ \\
\hline Sexo & Feminino & 316 & 47,8 \\
& Masculino & 345 & 52,2 \\
\hline Faixa Etária & $7-8$ anos & 90 & 13,7 \\
& 9-10 anos & 255 & 38,5 \\
& $11-12$ anos & 316 & 47,8 \\
\hline Tipo de Escola & Pública (4) & 355 & 53,7 \\
& Privada (2) & 306 & 46,3 \\
\hline \multirow{2}{*}{ Série } & $2^{\text {a }}$ Série & 82 & 12,4 \\
& $3^{\text {a }}$ Série & 92 & 13,9 \\
& $4^{\text {a }}$ Série & 156 & 23,6 \\
& $5^{\text {a } \text { Série }}$ & 180 & 27,2 \\
& $6^{a}$ Série & 151 & 22,8 \\
\hline Total & & 661 & 100,0 \\
\hline
\end{tabular}

\section{Resultados}

A análise dos itens da Escala de Afeto Positivo e Negativo para Crianças ocorreu em várias etapas. Primeiramente, realizou-se uma Análise Fatorial Exploratória que permitiu identificar entre quatro fatores que emergiram uma solução de dois fatores, os quais claramente identificavam o primeiro e segundo fatores como o afeto negativo e o positivo, respectivamente. Os fatores restantes agrupavam poucos itens de forma sem significado. A seguir, foi conduzida uma outra Análise Fatorial, através do Método dos Componentes Principais, utilizando a rotação Varimax, devido à baixa correlação entre os fatores, apontada pela literatura (Watson, Clark \& Tellegen, 1988), especificando dois fatores. Confirmou-se que a melhor solução fatorial, evidenciada pela literatura, foi a extração de dois fatores: um positivo e um negativo. Através desta análise, identificou-se que o item 21 ("emocionado") carregou nos dois fatores, sendo então eliminado. Uma nova análise foi realizada com os 43 itens da escala. A escala de afeto negativo apresentou 26 itens e a escala de afeto positivo 17. Optou-se, com a finalidade de reduzir o tamanho da escala e parear as subescalas, por excluir nove itens da escala de afeto negativo com as menores cargas fatoriais. Através deste procedimento, a Escala de Afeto Positivo e Negativo finalizou com 34 itens, 17 itens em cada subescala. Na Tabela 2, é apresentada a Matriz Fatorial dos Itens da Escala de Afeto Positivo e Negativo, composta pelos itens que permaneceram, agrupados pelas dimensões as quais pertencem, em ordem decrescente de suas cargas fatoriais. Além disso, é apresentado na Tabela 2 os valores dos Eigenvalues, os valores da variância explicada, do Alpha de Cronbach, da Média e do Desvio Padrão.

Observa-se que neste estudo, o primeiro fator extraído foi o negativo, enquanto em outros estudos foi o fator positivo (Watson, Clark \& Tellegen, 1988; Laurents \& cols., 1999). A amplitude das subescalas varia de 17 a 85 . As subescalas apresentaram elevados níveis de fidedignidade. O Alpha da Escala total foi de 0,90. A versão final da Escala de Afeto Positivo e Negativo é apresentada no ANEXO A.

Para investigar os efeitos do sexo, do tipo de escola e da faixa etária das crianças foi realizada uma 
Tabela 2. Matriz Fatorial da Escala de Afeto Positivo e Negativo

\begin{tabular}{|c|c|c|}
\hline \multirow{2}{*}{ Itens } & \multicolumn{2}{|c|}{ Fator } \\
\hline & 1 & 2 \\
\hline Irritado & 0,66 & \\
\hline Magoado & 0,65 & \\
\hline Perturbado & 0,63 & \\
\hline Nervoso & 0,63 & \\
\hline Triste & 0,63 & \\
\hline Furioso & 0,63 & \\
\hline Culpado & 0,60 & \\
\hline Preocupado & 0,60 & \\
\hline Chateado & 0,59 & \\
\hline Humilhado & 0,59 & \\
\hline Deprimido & 0,59 & \\
\hline Desanimado & 0,57 & \\
\hline Incomodado & 0,56 & \\
\hline Assustado & 0,55 & \\
\hline Amedrontado & 0,45 & \\
\hline Envergonhado & 0,44 & \\
\hline Impaciente & 0,42 & \\
\hline Divertido & & 0,69 \\
\hline Contente & & 0,67 \\
\hline Carinhoso & & 0,62 \\
\hline Feliz & & 0,61 \\
\hline Animado & & 0,61 \\
\hline Alegre & & 0,61 \\
\hline Participativo & & 0,56 \\
\hline Satisfeito & & 0,53 \\
\hline Esperto & & 0,52 \\
\hline Forte & & 0,51 \\
\hline Amoroso & & 0,51 \\
\hline Corajoso & & 0,47 \\
\hline Decidido & & 0,47 \\
\hline Esforçado & & 0,46 \\
\hline Cuidadoso & & 0,44 \\
\hline Delicado & & 0,38 \\
\hline Interessado & & 0,38 \\
\hline Eigenvalues & 6,48 & 4,61 \\
\hline \% Variância Explicada & 19,07 & 13,57 \\
\hline Alpha de Cronbach & 0,88 & 0,84 \\
\hline Média & 31,0 & 66,3 \\
\hline Desvio Padrão & 10,9 & 9,69 \\
\hline
\end{tabular}

Análise de Variância Multivariada (MANOVA) $2 \times 2 \times 3$, tendo como variáveis dependentes as duas subescalas de afeto. Não foram encontrados efeitos de interação tanto para Afeto Positivo $[F(2,621)=1,24 ; p<0,29]$ quanto para Afeto Negativo $[F(2,62 I)=1,20$; $p<0,30]$. Foram identificados efeitos principais para sexo $[F(I, 62 I)=5,15 ; p<0,02]$ e faixa etária $[F(2,62 I)=9,02 ; p<0,0 I]$ somente na subescala de Afeto Positivo. Quanto ao sexo, observou-se que as meninas $(M=3,93 ; D P=0,60)$ reportaram menores 
níveis de afeto positivo do que os meninos $(M=4,03$; $D P=0,59)$. Não foram encontradas diferenças significativas quanto ao afeto negativo. Quanto à faixa etária, através do Teste para Comparação de Médias a posteriori, Tukey, foram constatadas diferenças significativas entre as crianças de 7-8 anos $(M=4,20$; $D P=0,56 ; p=0,0 \mathrm{I})$, que relataram maiores níveis de afeto positivo, e as crianças de $9-10$ anos $(M=3,99$; $D P=0,59)$ e as crianças de $\mathrm{II}-\mathrm{I} 2$ anos $(M=3,91 ; D P=0,59)$, que reportaram menores níveis de afeto positivo.

\section{Estudo 2}

\section{Estudo de Validação Concorrente da Escala de Afeto Positivo e Negativo para Crianças}

\section{Método}

\section{Participantes}

Para a realização do estudo de validação concorrente participaram 230 crianças de ambos os sexos, II 7 meninos $(50,9 \%)$ e II3 meninas $(49,18 \%)$. A faixa etária variou entre oito e 12 anos $(M=10,6$ anos; $D P=1,7$ anos $)$. As crianças freqüentavam o ensino fundamental (de $3^{\mathrm{a}}$ a $6^{\mathrm{a}}$ série) de escolas públicas estaduais $(57,4 \%)$ e privadas $(42,6 \%)$ de Porto Alegre.

\section{Instrumentos}

Para avaliar as variáveis correlatas foram eleitas as variáveis relativas à auto-estima, ansiedade e depressão, tendo em vista que em alguns estudos encontraram-se correlações significativas dessas com o afeto positivo e negativo (Laurent e cols., 1999), assim como com a satisfação de vida global e multidimensional (Giacomoni, 2002), outros componentes do bem-estar subjetivo.

Para avaliar auto-estima foi utilizada a versão adaptada para o português (Hutz, 2000) da Escala de AutoEstima de Rosenberg (1965). Esse instrumento é uma escala de auto-relato do tipo Likert (quatro pontos I4) composta originalmente por dez itens que investigam aspectos globais da auto-estima. A escala foi desenvolvida para adolescentes, sendo bastante difundida devido à sua praticidade de aplicação, entretanto, sua aplicação em crianças a partir de oito anos de idade tem sido comum (Houtte \& Jarvis, 1995). A versão adaptada da escala adicionou mais um item, mantendo-se a estrutura unidimensional da mesma (Hutz, 2000). Os participantes devem indicar o grau de concordância com o item descrito. Quanto maior o escore obtido, maior a auto-estima. A escala tem apresentado índices de fidedignidade constantes e aceitáveis para uso em pesquisa (acima de 0,80). A pesquisa realizada com a escala adaptada demonstrou parâmetros psicométricos apropriados (Hutz, 2000). No presente estudo de validação da Escala de Afeto, a Escala de Auto-Estima apresentou um índice de consistência interno de 0,75 (Alpha de Cronbach).

A ansiedade foi medida utilizando-se o IDATE - C (Spielberger, 1983), Inventário de Ansiedade TraçoEstado, já adaptado e padronizado para o seu uso no Brasil por Biaggio (1980). O IDATE-C foi desenvolvido a partir do Inventário de Ansiedade Traço-Estado na forma adulta (Spielberger, Gorsuch \& Lushene, 1979; Biaggio, Natalício \& Spielberger, 1977). A forma infantil é composta por duas escalas do tipo auto-relato que visam medir a ansiedade-estado (FORMA C-I) e a ansiedade-traço (FORMA C-2), dois conceitos distintos de ansiedade. Utilizada com crianças de ensino fundamental, essa escala é composta por 20 itens em cada subescala, podendo ser aplicada de forma coletiva. Neste estudo, os valores encontrados quanto à fidedignidade foram satisfatórios. Para forma C-I foi encontrado um Alpha de Cronbach de 0,84, para a forma C-2, Alfa de Cronbach de 0,73.

Para avaliar a depressão, foi utilizado o Children's Depression Inventory (CDI) elaborado por Kovacs (1983, 1992), a partir do Beck Depression Inventory para adultos. O objetivo do CDI é detectar a presença e a severidade do transtorno depressivo na infância. Destina-se a identificar alterações afetivas em crianças e adolescentes dos sete aos dezessete anos. É uma medida unifatorial composta por 27 itens, cada um com três opções de resposta (pontuada como 0, I ou 2), na qual a criança deve assinalar a que melhor descreve o seu estado nos últimos tempos. O CDI pode ser aplicado tanto individualmente quanto coletivamente. Em relação às propriedades 
psicométricas da escala, a consistência interna descrita por Kovacs (I980/I98I) mostrou-se adequada $(0,86)$. Kovacs estabeleceu como ponto de corte do CDI o escore 19. No Brasil, o CDI foi adaptado por Hutz e Giacomoni (2000). As pesquisas que utilizaram versões adaptadas para o contexto brasileiro vêm apresentando condições psicométricas adequadas. $\mathrm{O}$ Alpha de Cronbach do inventário adaptado para o uso no nordeste do país por Gouveia, Barbosa, Almeida e Gaião (1995) foi de 0,8I. Em pesquisas realizadas com amostras infantis no Rio Grande do Sul, observaramse os seguintes coeficientes de Alpha de Cronbach: 0,80 (Giacomoni, 1998) e 0,92 (Reppold, 200I). Outros estudos que avaliaram crianças e adolescentes do Rio Grande do Sul em situação de risco obtiveram um Alpha de até 0,79 (Dell'Aglio, 2000; Silva, 200I). No presente estudo, foi encontrado um Alpha de Cronbach de $0,8 \mathrm{I}$. Cabe ressaltar que faltam estudos brasileiros sobre o ponto de corte da escala.

A satisfação de vida, que pode ser avaliada da vida como um todo ou sobre domínios específicos (por exemplo: escola, família, amizade), foi medida através dois instrumentos construídos e desenvolvidos especialmente em nosso meio: Escala de Satisfação de Vida Global Infantil e Escala Multidimensional de Satisfação de Vida para Crianças (Giacomoni, 2002). A Escala de Satisfação de Vida Global Infantil é um instrumento unidimensional de tipo likert (5 pontos) composto por 7 itens que avalia a satisfação de vida de forma global (por exemplo: "estou satisfeito com a minha vida"). A Escala Multidimensional de Satisfação de Vida para crianças possui com 50 itens, distribuídos em seis fatores: Self, Self Comparado, Não-Violência, Família, Amizade e Escola. Foram encontradas consistências internas adequadas para cada uma das escalas, Alfa de Cronbach acima de 0,80 . Os estudos de construção e validação das escalas foram submetidos para publicação.

\section{Procedimento}

As escolas foram contatadas para a apresentação do projeto e para o encaminhamento dos termos de consentimento informado aos pais e/ou responsáveis. As escalas foram aplicadas de forma coletiva em sala de aula durante um único encontro. A ordem de aplicação dos instrumentos foi aleatória.

\section{Resultados}

Para a verificação da validade concorrente da Escala de Afeto Positivo e Negativo foram examinados os padrões de correlações entre as suas subescalas e a Escala de Auto-Estima, o Inventário de Depressão Infantil (CDI), as Subescalas do Inventário de Ansiedade Traço-Estado (IDATE-C FORMA CI-Estado, FORMA C2-Traço) e as Medidas de Satisfação de Vida desenvolvidas por Giacomoni (2002) (Escala de Satisfação de Vida Global Infantil e Escala Multidimensional de Satisfação de Vida para Crianças). A Tabela 3 apresenta as correlações entre as Escalas de Afeto e a Escala de Satisfação de Vida Global e os fatores da Escala Multidimensional de Satisfação de Vida. A Tabela 4 apresenta as correlações entre as subescalas de afeto e entre os instrumentos que avaliaram auto-estima, depressão e ansiedade.

A correlação encontrada entre Afeto Positivo e Negativo foi relativamente pequena, de $-0,15$ ( $n=$ 654; $p<0,01$ ). Esse valor de correlação entre as subescalas de Afeto Positivo e Afeto Negativo é consistente com os resultados reportados por Watson, Clark e Tellegen (1988) no estudo de construção da PANAS para adultos $(r=-0,08)$ e é equivalente ao encontrado por Laurent e colaboradores (1999) no estudo de construção da versão infantil da PANAS $(r=-0,16)$. Além disso, as correlações obtidas entre a Escala de Afeto Positivo e Afeto Negativo e as medidas de Satisfação de Vida Global e com os Domínios reforçam as evidências de validade concorrente, uma vez que se esperaria uma correlação positiva de média à elevada entre as medidas de afeto positivo e satisfação e uma correlação negativa média entre afeto negativo e satisfação de vida. Observa-se que entre os domínios, - Self, a Família e a Amizade despontam entre os principais domínios de afeto positivo. Novamente encontramos evidências de que as relações interpessoais e os recursos do Self estão entre os maiores promotores de bem-estar subjetivo. As correlações entre as medidas de desajustamento emocional, como depressão e ansiedade e a medida de afeto negativo foram nas magnitudes e sentido 
Tabela 3. Matriz de Correlação entre as Subescalas de Afeto Positivo e Negativo e as Subescalas de Satisfação de Vida Multidimensional e a Escala de Satisfação de Vida Global

\begin{tabular}{lcc}
\hline Subescalas & Afeto Positivo & Afeto Negativo \\
\hline 1. Self & $0,67^{* *}$ & $-0,28^{* *}$ \\
& $n=644$ & $n=644$ \\
2. Self_Comparado & $0,25^{* *}$ & $-0,39 * *$ \\
& $n=636$ & $n=636$ \\
3. Não-Violência & $0,14^{* *}$ & $-0,39 * *$ \\
& $n=643$ & $n=643$ \\
4. Família & $0,51^{* *}$ & $-0,27 * *$ \\
& $n=645$ & $n=645$ \\
5. Amizade & $0,50^{* *}$ & $-0,34 * *$ \\
& $n=641$ & $n=641$ \\
6. Escola & $0,49^{* *}$ & $-0,25^{* *}$ \\
Satisfação Global de Vida & $n=648$ & $n=648$ \\
& $0,59^{* *}$ & $-0,31^{* *}$ \\
& $n=655$ & $n=655$ \\
\hline
\end{tabular}

*** $p<0,01$

Tabela 4. Matriz de Correlação entre as Subescalas de Afeto Positivo e Negativo e os Escores de Auto-Estima, Depressão (CDI), Ansiedade-Estado (IDATECI) e Ansiedade-Traço (IDATE-C2)

\begin{tabular}{lcc}
\hline Escalas & Afeto Positivo & Afeto Negativo \\
\hline Auto-Estima & $0,48^{* *}$ & $-0,35^{* *}$ \\
& $n=229$ & $n=229$ \\
CDI & $-0,40^{* *}$ & $0,41^{* *}$ \\
& $n=216$ & $n=216$ \\
IDATE C1 & $-0,51^{* *}$ & $0,49 * *$ \\
Estado & $n=224$ & $n=224$ \\
IDATE C2 & $-0,24^{* *}$ & $0,39 * *$ \\
Traço & $n=217$ & $n=217$ \\
\hline$* * 0<0$ I & &
\end{tabular}

esperados. Laurent e seus colaboradores (1999) encontraram uma correlação de 0,59 entre o CDI e a subescala de afeto negativo da PANAS - $\mathrm{C}$ e uma correlação de 0,68 entre a subescala de ansiedadetraço e a subescala de afeto negativo. $O$ mesmo ocorreu entre a medida de auto-estima e afeto positivo.

\section{Considerações Finais}

Foram apresentadas informações sobre o desenvolvimento de escalas curtas que medem os dois aspectos primários do afeto - o positivo e o negativo - componentes do bem-estar subjetivo infantil. A Escala de Afeto Positivo e Negativo para crianças demonstrou 
características psicométricas adequadas muito parecidas as da PANAS na versão infantil (Laurent \& cols., 1999). Neste estudo, os coeficientes Alpha obtidos, foram para a subescala de Afeto Positivo foi 0,88 e de 0,84 para a subescala de Afeto Negativo, sendo considerados satisfatório. Laurent e seus colaboradores (1999) reportaram coeficientes de Alpha de 0,90 para Afeto Positivo e 0,92 para Afeto Negativo. Também foi obtida uma solução de dois fatores em ambos estudos. A baixa correlação entre os fatores reforça a evidência da independência dos mesmos. Pode-se indicar através deste estudo indícios de validade de construto da escala.

Além das características psicométricas adequadas, a Escala apresentou boas evidências de validação concorrente. Através de correlações coerentes com as apontadas pela literatura, os resultados indicam que a Escala de Afeto Negativo pode ser utilizada como instrumento de avaliação de desajustamento emocional. Quanto ao afeto positivo, observou-se uma correlação média entre esse e auto-estima $(0,48)$. Indica-se a necessidade de uma maior investigação deste componente do afeto junto a outras variáveis do Self, como, por exemplo, capacidade de socialização e auto-eficácia. Observa-se na literatura uma ausência de estudos de validação para essa medida positiva.

Os resultados referentes às diferenças de gênero quanto ao afeto, indicaram que as meninas apresentam menores níveis de afeto positivo. Esse resultado não é corroborado pela literatura que aponta que as mulheres apresentam maiores índices de felicidade (Nolen-Hoeksema \& Rusting, 1999; Nolen-Hoeksema \& Girgus, 1994), no entanto, cabe ressaltar que são praticamente inexistentes estudos sobre afeto de crianças. Não foi confirmada a premissa teórica de que meninas apresentam maiores níveis de afeto negativo (Fujita, Diener \& Sandvik, 199I). Malatesta e colaboradores (1986) relataram uma diminuição de afeto negativo e um aumento linear de afeto positivo em crianças entre dois anos e meio e sete anos. Os resultados, pelo contrário, apontaram uma baixa de afeto positivo ao longo das faixas etárias, fato que não havia sido confirmado em estudos com amostras infantis até então. No entanto, Diener e Suh (1998) relataram, em seu grande estudo transcultural, essa tendência entre adultos. Não foram encontradas diferenças quanto ao tipo de escola, indicando uma possível ausência da importância dos aspectos sócioeconômicos nessa avaliação.

Os valores de correlação de médio a elevados entre a subescala de afeto positivo e as medidas de satisfação de vida também proporcionaram mais evidências de validade para ambas as medidas de bemestar subjetivo. Entretanto, cabe indicar a necessidade de maiores estudos para completar o processo de validação das escalas. Também caberiam maiores investigações sobre a natureza dos fatores. A literatura apresenta de forma bastante ampla os aspectos relacionados ao afeto negativo, mas a lacuna é ainda maior quando se trata do afeto positivo.

São reconhecidas algumas limitações, quanto à amostra e ao método no que tange ao presente estudo. A amostra deve ser ampliada para grupos clínicos e para outras regiões do país, além de outras realidades sócio-econômicas e culturais. Quanto aos tipos de medidas, uma ressalva deve ser feita para o fato de que foram utilizadas somente medidas de auto-relato, indicando-se a ampliação das técnicas de avaliação para proporcionar maior validade. Além disso, novos estudos com replicação devem ser conduzidos para também promoverem novas formas de validação.

O desenvolvimento da Escala de Afeto Positivo e Negativo para Crianças incrementa o processo de elaboração de instrumentos para avaliarmos o bemestar subjetivo infantil. Os resultados encontrados indicam que as escalas serão de grande utilidade para o desenvolvimento da área de estudos do bem-estar subjetivo infantil, incentivando o prosseguimento de novas pesquisas.

\section{Referências}

Andrews, F. M. \& Whitey, S. B. (1976). Social indicators of wellbeing: America's perception of quality of life. New York, NY: Plenum Press.

Biaggio, A. M. B. (1980). Desenvolvimento da forma infantil em português do inventário de ansiedade traço-estado 
de Spielberger. Arquivos Brasileiros de Psicologia Aplicada, 32, $106-118$.

Biaggio, A. M. B., Natalício, L. \& Spielberger, C. D. (1977). Desenvolvimento da forma experimental em português do inventário de ansiedade traço-estado (IDATE) de Spielberger. Arquivos Brasileiros de Psicologia Aplicada, 29, $3 \mid-44$

Campbell, A., Converse, P. \& Rodgers, W. L. (1976). The quality of American life: perceptions, evaluations, and satisfactions. New York,NY: Russell Sage Foundation.

Cozby, P. (198I). Methods in behavioral research. Califórnia: Mayfield Publishing.

Dell'Aglio, D. D. (2000). O processo de coping, institucionalização e eventos de vida em crianças e adolescentes. Tese de Doutorado não publicada, Curso de Pós-Graduação em Psicologia do Desenvolvimento, Universidade Federal do Rio Grande do Sul. Porto Alegre, RS.

Diener, E. (1994). Assessing subjective well-being: progress and opportunities. Social Indicators Research, 3I, I03-I 57.

Diener, E. (1 984). Subjective well-being. Psychological Bulletin, $95,542-575$.

Diener, E., Lucas, R. E. \& Oishi, S. (2005). Subjetive well-being: the science of happiness and life satisfaction. Em C. R. Snyder \& S. J. Lopez (Eds.), Handbook of Positive Psychology (63-73) New York,NY: Oxford University Press.

Diener, E. \& Suh, E. (1998). Age and subjective well-being: an international analysis. Annual Review of Gerontology and Geriatrics, I7, 304-324.

Diener, E., Suh, E. M., Lucas, R. E. \& Smith, H. L. (1999). Subjective well-being: Three decades of Progress. Psychological Bulletin, I25, 276-302.

Fujita, F., Diener, E. \& Sandvik, E. (1991). Gender differences in negative affect and well-being: The case for emotional intensity. Journal of Personality and Social Psychology, 6I, 427-34.

Giacomoni, C. H. (1998). Desempenho Escolar, Controle Percebido e Eventos de Vida como Preditores de Bem-Estar Subjetivo em Crianças. Dissertação de Mestrado não publicada. Curso de Pós-Graduação em Psicologia do Desenvolvimento, Universidade Federal do Rio Grande do Sul. Porto Alegre, RS.

Giacomoni, C. H. (2002). Bem-estar Subjetivo Infantil: conceito de felicidade e construção de instrumentos para avaliação.
Tese de Doutorado não publicada. Curso de Pós-Graduação em Psicologia do Desenvolvimento, Universidade Federal do Rio Grande do Sul. Porto Alegre, RS.

Giacomoni, C. H. \& Hutz, C. S. (1997). A mensuração do bem-estar subjetivo: escala de afeto positivo e negativo e escala de satisfação de vida [Resumos]. Em Sociedade Interamericana de Psicologia (Org.), Anais XXVI Congresso Interamericano de Psicologia (p. 3 | 3). São Paulo, SP: SIP.

Gouveia, V. V., Barbosa, G. A., Almeida, H. J. F. \& Gaião, A. A. (1995). Inventário de Depressão Infantil - CDI: estudo de adaptação com escolares de João Pessoa. Jornal Brasileiro de Psiquiatria, 44, 345-349.

Houtte, B. A. \& Jarvis, P. A. (1995). The role of pets in preadolescent psychological development. Journal of Applied Developmental Psychology, 16, 463-479.

Hutz, C. S. (2000). Adaptação brasileira da escala de auto-estima de Rosenberg. Manuscrito não publicado, Curso de PósGraduação em Psicologia do Desenvolvimento, Universidade Federal do Rio Grande do Sul. Porto Alegre, RS.

Hutz, C. S. \& Giacomoni, C. H. (2000). Adaptação brasileira do inventário de depressão infantil $(C D I)$. Manuscrito não publicado, Curso de Pós-Graduação em Psicologia do Desenvolvimento, Universidade Federal do Rio Grande do Sul. Porto Alegre, RS.

Kovacs, M. (1992). Children's Depression Inventory Manual. Los Angels,CA: Western Psychological Services.

Kovacs, M. (1983). The Children's Depression Inventory: A SelfRated Depression Scale for School Age Youngsters. Pittsburg, PA: University of Pittsburgh, School of Medicine.

Kovacs, M. (1980/198I). Rating scales to assess depression in school-aged children. Acta Paedopsychiatrica, 46, 305-316.

Laurent, J., Catanzaro, S. J., Joiner, T.E., Rudolph, K.D., Potter, K. I., Lambert, S., Osborne, L. \& Gathright, T. (1999). A measure of positive and negative affect for children: Scale development and preliminary validation. Psychological Assessment, II, 326-338.

Lucas, R. E., Diener, E. \& Larsen, R. J. (2003). Measuring Positive Emotions. Em S. J. Lopez \& C. R. Snyder (Eds.) Positive Psychological Assessment: A handbook of models and measures. (20l-2l8).Washington,DC: American Psychological Association.

Malatesta, C. Z., Grigoryev, P., Lamb, C., Albin, M. \& Culver, C. (1986). Emotion socialization and expressive 
development in pre-term and full-term infants. Child Development, 57, 316-30.

Nolen-Hoeksema, S. \& Rusting, C. L. (1999). Gender differences in well-being. Em D. Kahneman, E. Diener \& N. Schwarz (Eds). Well-being: The foundations of hedonic psychology (pp. 330-344). New York, NY: Russell Sage Foundation.

Nolen-Hoeksema, S. \& Girgus, J. S. (1994). The emergence of gender differences in depression during adolescence. Psychological Bulletin, I/5, 424-43.

Reppold, C. T. (200I). Estilo parental percebido e adaptação psicológica de adolescentes adotados. Dissertação de Mestrado não publicada. Curso de Pós-Graduação em Psicologia do Desenvolvimento, Universidade Federal do Rio Grande do Sul. Porto Alegre, RS.

Rosenberg, M. (1965). Society and the adolescent self-image. Princeton,NJ: Princeton University Press.

Silva, D. F. M. (200I). O desenvolvimento das trajetórias do comportamento delinqüente em adolescentes infratores.
Manuscrito não publicado, Curso de Pós-Graduação em Psicologia do Desenvolvimento, Universidade Federal do Rio Grande do Sul. Porto Alegre, RS.

Spielberger, C. D. (1983). Manual do inventário de ansiedade traço-estado-C. Rio de Janeiro, RJ:CEPA.

Spielberger, C. D., Gorsuch, R. L. \& Lushene, R. E. (1979). Manual do inventário de ansiedade traço-estado. Rio de Janeiro,RJ: CEPA.

Watson, D., Clark, L.A. \& Tellegen, A. (1988). Development and validation of brief measures of positive and negative affect: The PANAS Scales. Journal of Personality and Social Psychology, 69, 719-727.

Recebido em: 22/08/2006

Revisado em: 30/I I/2006

Aprovado em: 19/12/2006

Claudia Hofheinz Giacomoni (giacomon@uol.com.br) é doutora pelo Curso de Pós-Graduação em Psicologia do Desenvolvimento da UFRGS e Professora do Curso de Psicologia da Universidade Federal de Santa Maria.

Cláudio Simon Hutz é doutor pela University of lowa, EUA e Professor do Programa de Pós-graduação stricto Sensu em Psicologia do Desenvolvimento do Instituto de Psicologia da Universidade Federal do Rio Grande do Sul.

Endereço para correspondência

Claudia H. Giacomoni

UFSM Rua Floriano Peixoto, 1750 sala 307

97015-372 - Santa Maria, RS 